\title{
Ausencia de asociación entre síndrome metabólico y síntomas depresivos en adultos colombianos
}

\author{
Luis D íaz-Martínez, Norma Serrano, Juan Pinzón, \\ Gerardo Mantillaa, Harvy Velasco, Luz Martínez, \\ Paula Millán, Sandra Acevedo, Daniel Moreno, Sara E Sus ${ }^{b}$. \\ Lack of association between \\ metabolic syndrome and depressive \\ symptoms in Colombian adults
}

Background: There is a possible association depressive symptoms (DS) and metabolic syndrome (MS), to the extent that treating one condition improves the other. Aim: To estimate the association between MS and DS among the employees of a medical school. Material and methods: Cross sectional study of 159 people aged $41 \pm 11$ years (88 men). MS was evaluated according to International Diabetes Federation (IDF), National Cholesterol Education Program (NCEP)/Adult Treatment Panel III (ATP-III) and ATP-IIIa criteria and the depression questionnaire of the Center for Epidemiological Studies (CESD) was used for DS. A multivariate logistic regression was performed adjusting for age and gender. Results: The prevalence of MS according to ATP-III was 13.2\% (95\% CI: 8.4-19.5), to ATP-IIIa was 34.0\% (95\% CI: 26.6-41.9) and to IDF was 33.3\% (95\% CI: 26.1-41.2). The prevalence of clinically relevant SD was $15.1 \%$ (95\% CI: 9.9-21.6). No significant association was found between DS and MS according to the different criteria: ATP-III OR 1.30 (95\% CI: 0.40-4.24), ATP-IIIa OR 0.94 (95\% CI: 0.37-2.33), IDF OR 1.20 (95\% CI: 0.49-2.95). Conclusions: In this series, no association was observed between MS and depression (Rev Méd Chile 2007; 135: 990-6).

(Key words: Brain diseases, metabolic; Depression; Metabolic syndrome X)

\author{
Recibido el 21 de diciembre, 2006. Aceptado el 28 de febrero, 2007. \\ Investigación financiada por la Dirección General de Investigaciones de la Universidad \\ Autónoma de Bucaramanga, Colombia (Proyecto EGEN 18). \\ Centro de Investigaciones Biomédicas, Facultad de Medicina, Universidad Autónoma de \\ Bucaramanga, Bucaramanga, Colombia. \\ aLaboratorista Clínico. MSc \\ bEstudiante, Facultad de Medicina, Universidad Autónoma de Bucaramanga, \\ Bucaramanga, Colombia.
}

\footnotetext{
Correspondencia a: Dr. Luis Díaz. Centro de Investigaciones Biomédicas, Facultad de Medicina, Universidad Autónoma de Bucaramanga. Calle 157 \#19-55, Bucaramanga, Colombia. Fax: 6399147. Teléfono: 6399156. E mail: genesis@unab.edu.co
} 
$\mathrm{E}^{\mathrm{n}}$ n todo el mundo, los trastornos neuropsiquiátricos son la principal causa de incapacidad, con $37 \%$ de los años perdidos por incapacidad entre las personas de 15 o más años; entre ellos, $50 \%$ corresponde a trastorno depresivo mayor $(\mathrm{TDM})^{1}$. Esto se corresponde con los datos nacionales y locales, ya que en Colombia, la prevalencia estimada de TDM en el último año es de $10,0 \%^{2}$, mientras que la prevalencia entre los habitantes de Bucaramanga es 16,5\%3.

Por otro lado, en Latinoamérica y el Caribe, la enfermedad cardiovascular, junto con la diabetes mellitus, fueron las responsables de $34 \%$ de todas las muertes que se presentaron en $2000^{4}$, y se espera para el futuro un incremento en su incidencia, secundario al envejecimiento de la población y de la alta prevalencia de los factores de riesgo cardiovascular (hipertensión, obesidad, diabetes y dislipidemia) ${ }^{5}$.

El síndrome metabólico (SM) es una constelación de signos y resultados de laboratorio (obesidad abdominal, hipertrigliceridemia, bajo nivel de colesterol HDL, hipertensión arterial e hiperglicemia), el cual es reconocido como factor de riesgo para diabetes, infarto agudo del miocardio y enfermedad cerebrovascular ${ }^{6}$. Existen varias definiciones de SM, siendo las más conocidas las del National Cholesterol Education Program (NCEP)/ Adult Treatment Panel III (ATP-III) ${ }^{7}$, la de la International Diabetes Federation (IDF) ${ }^{8}$ y la definición actualizada del ATP-III (ATP-IIIa) ${ }^{9}$. Dependiendo de la definición utilizada, en los países del norte de Sudamérica la prevalencia de SM oscila entre $23,6 \%$ y $33,0 \% 10-12$.

Ahora bien, se ha encontrado asociación entre resistencia a la insulina, el aspecto nuclear del SM y $\mathrm{TDM}^{13}$, particularmente en mujeres, entre quienes hay un exceso de riesgo de desarrollar SM, dado que presentan depresión del orden de $29 \%$ (IC 95\% 3\%-62\%) por cada año, comparado con las mujeres que no lo están ${ }^{14,15}$.

Esto ha conducido a varios estudios en los que se ha documentado asociación entre TDM con diferentes tipos de enfermedad cardiovascular, tanto con su desarrollo como con su progresión ${ }^{16,17}$, es más, la reducción del índice de masa corporal, reflejo de la obesidad (uno de los elementos que conforman el SM), se traduce en mejoría de las alteraciones del ánimo, independientemente de los cambios del aspecto físico ${ }^{18}$.
Esto soporta hallazgos previos que indican que la asociación entre SM y TDM es de naturaleza medioambiental, no sólo genética ${ }^{19}$ y que la intervención de uno de los dos problemas tendría consecuencias favorables sobre la severidad del otro $^{20}$.

El objetivo de este informe es evaluar la fuerza de la asociación que pudiera existir entre síntomas depresivos (SD) y SM en una población de empleados (profesores y personal administrativo) de la Facultad de Medicina de la Universidad Autónoma de Bucaramanga (UNAB), Colombia.

\section{MATERIAL Y MÉTODO}

GENESIS es un estudio transversal realizado entre los empleados de la Facultad de Medicina de la Universidad Autónoma de Bucaramanga, que busca identificar factores de riesgo convencionales y genéticos asociados con SM y su correlación con fenotipos intermedios. El estudio fue aprobado previamente por el Comité de Ética en Investigación de la Facultad de Medicina de la UNAB. Los participantes dieron su consentimiento informado por escrito.

Todos los empleados de la Facultad de Medicina fueron invitados a participar; asistieron a cita médica en donde se les realizó entrevista clínica, examen físico y toma de pruebas de laboratorio a fin de establecer los criterios de las tres definiciones de $\mathrm{SM}^{7-9}$. No se incluyeron personas que en el momento del estudio tuvieran enfermedad crónica grave (v. gr. insuficiencia hepática, insuficiencia renal, cáncer, diabetes mellitus tipo 1), infecciones sistémicas o estuvieran embarazadas.

En la entrevista clínica se preguntó sobre aspectos demográficos, antecedentes personales y familiares de riesgo cardiovascular, tanto tradicionales como no convencionales. Inmediatamente se aplicó de manera autodiligenciada el Cuestionario para Depresión del Centro de Estudios Epidemiológicos (CES-D). Esta escala es utilizada para la detección precoz de SD de importancia clínica; ha sido validada en población adulta de Bucaramanga, donde se encontró que la prueba tiene en esta población un área bajo la curva receptor operador de 0,904 y un alfa de Cronbach de 0,865; mientras que para un puntaje de 180 más, la sensibilidad del cuestionario es de 97,7\% y 
la especificidad de $67,7 \%$ (observaciones no publicadas).

Durante el examen físico se realizaron dos tomas de tensión arterial, peso, talla y de perímetro de cintura ${ }^{21,22}$. Las pruebas de laboratorio realizadas fueron glicemia en ayunas, colesterol total, colesterol HDL y triglicéridos. En aquellas personas con glicemia en ayunas entre 100-125 mg/dL se realizó glicemia poscarga a las dos horas, para establecer si los pacientes eran además intolerantes a la glucosa o diabéticos tipo 2. La glicemia se midió con el método de la glucosa-oxidasa; el colesterol total por la técnica de la colesterol éster hidrolasa-colesterol oxidasa; el colesterol HDL se determinó precipitando el suero con ácido fosfotúngstico y cuantificado con la misma técnica del colesterol total; los triacilgliceroles se analizaron por la técnica de la glicerol fosfato oxidasa y la glicerol cinasa.

La presencia de SM en cada participante se estableció aplicando los criterios de las tres definiciones existentes (ATP-III, ATP-IIIa e IDF); en los tres casos se utilizó como límite de perímetro abdominal el propuesto en la IDF para suramericanos: $90 \mathrm{~cm}$ para varones y $80 \mathrm{~cm}$ para mujeres ${ }^{8}$.

Los datos obtenidos de la población estudiada se describieron con tablas y proporciones, incluyendo su intervalo de confianza de 95\% (IC 95\%) para las variables nominales $\mathrm{u}$ ordinales; las variables discretas se analizaron con promedio y desviación estándar (DE), o mediana y recorrido intercuartil (RIQ), según la distribución, fuese normal o no ${ }^{23}$. Para estimar la asociación entre SM y SD relevantes, la presencia de SM, según cada definición, se comparó tanto con el puntaje absoluto del CES-D como con la presencia o no de TDM dado un puntaje de 18 o más; las diferencias en el primer caso se evaluaron por medio de la prueba de suma de rangos de Wilcoxon ${ }^{24}$, mientras que en la segunda se utilizó tanto la prueba $\chi^{2}$ y la estimación de la razón de disparidades (OR, de su nombre en inglés odds ratio ${ }^{25}$ ). Los OR se ajustaron teniendo en cuenta edad y sexo de los participantes por medio de regresión logística. Se consideraron significativas aquellas pruebas con $p<0,05$. Todos los procesos se hicieron con STATA 9,26.

\section{RESUltados}

De las 167 personas empleadas en la facultad, 159 aceptaron participar en el estudio; 55,4\% eran hombres (88 personas) y 44,6\% mujeres (71 personas). La edad osciló entre 22 y 73 años, con promedio de 40,8 años (desviación estándar [DE] 10,5), siendo las mujeres más jóvenes que los hombres (36,6 años [DE 9,6] vs 44,2 años [DE 10,1] $p<0,001$ ).

En la Tabla 1 se encuentra la proporción de participantes que cumplieron cada uno de los criterios de SM según ATP-III. La prevalencia de SM según cada definición osciló entre 13,2\% (ATPIII) y $34,0 \%$ (ATP-IIIa 34,0\%), con diferencias significativas entre hombres y mujeres, independientemente de la definición utilizada, así como entre la población de más de 40 años (Tabla 2).

El puntaje del CESD osciló entre 0 y 37 puntos, con mediana de 7 puntos y RIQ entre 4 y 14 puntos. $\mathrm{Si}$ se considera como de alto riesgo de tener síndrome depresivo mayor a quienes puntúan 18 o más, se encontró que 23 de las personas estaban afectadas (prevalencia de 15,1\%, IC 95\% 9,9-21,6). No hubo diferencias en el puntaje de los hombres (mediana en 7 puntos, RIQ entre 4 y 12) y el de las mujeres

\section{Tabla 1. Prevalencia de cumplimiento de los criterios para síndrome metabólico, según AT P-III entre la población estudiada}

\begin{tabular}{|ll|}
\hline Criterio & Personas (\%) \\
\hline Obesidad abdominal & $84(52,8 \%)$ \\
HTA $(\geq 130 / 85 \mathrm{~mm}$ Hg) & $18(11,3 \%)$ \\
TGD $\geq 150 \mathrm{mg} / \mathrm{dL}$ & $55(34,6 \%)$ \\
Glicemia $(\geq 100 \mathrm{mg} / \mathrm{dL})$ & $3(1,9 \%)$ \\
HDL $(<40 \mathrm{mg} / \mathrm{dL}$ varones, $<50 \mathrm{mg} /$ dL mujeres) & $152(95,6 \%)$ \\
\hline
\end{tabular}

HTA: Hipertensión arterial; TGD: triglicéridos; HDL: lipoproteínas de alta densidad. 
Tabla 2. Prevalencia de síndrome metabólico entre la población estudiada y por sexo o edad,
según las tres definiciones utilizadas

\begin{tabular}{|llccc|}
\hline \multirow{2}{*}{ Característica } & \multicolumn{3}{c|}{ Definición } \\
\cline { 2 - 5 } Toda la población & ATP-III (IC 95\%) & ATP-IIIa (IC 95\%) & IDF (IC 95\%) \\
\hline \multirow{2}{*}{ Sexo } & Hombres $(\mathrm{n}=88)$ & $21,8 \%(13,5-31,6)$ & $55,2 \%(44,7-66,3)$ & $56,3 \%(45,8-67,3)$ \\
& Mujeres $(\mathrm{n}=71)$ & $2,8 \%(0,3-9,8)$ & $8,5 \%(3,2-17,5)$ & $5,6 \%(1,6-13,8)$ \\
& (Valor p) & $(0,001)$ & $(<0,001)$ & $(<0,001)$ \\
\multirow{2}{*}{ Edad } & $\leq 40$ años $(\mathrm{n}=84)$ & $18,9 \%(11,3-29,1)$ & $47,3 \%(36,6-58,8)$ & $44,6 \%(33,2-55,3)$ \\
& $>40$ años $(\mathrm{n}=75)$ & $8,3 \%(3,0-16,6)$ & $22,6 \%(13,8-33,8)$ & $23,8 \%(14,9-35,3)$ \\
& (Valor p) & $(0,050)$ & $(<0,001)$ & $(0,006)$ \\
\hline
\end{tabular}

(mediana en 8 puntos, RIQ entre 4 y 17; $p=0,197$ ), ni entre las personas de 40 o más años (mediana 7 puntos, RIQ entre 3 y 12) frente a los más jóvenes (mediana 7 puntos, RIQ entre 4 y 14; $p=0,324$ ).

No hubo diferencia en el puntaje de la prueba CES-D entre las personas que tenían SM según ATPIII (mediana 7 puntos, RIQ entre 6 y 17 puntos) frente a las que no lo padecían (mediana 7 puntos, RIQ entre 4 y 13 puntos), tal como se aprecia en la Figura 1, panel superior ( $p=0,396)$. Igual fenómeno ocurrió cuando se utilizó la definición de ATP-IIIa (mediana de 7 puntos [RIQ 4 a 13 puntos] entre los positivos vs 7 puntos [RIQ 4 a 14 puntos] entre los negativos, $p=0,988$; Figura 2, panel medio) o la de la IDF (mediana de 7 puntos [RIQ 4 a 14 puntos] entre los positivos vs 7 puntos [RIQ 4 a 13 puntos] entre los negativos, $p=0,937$; Figura 1, panel inferior).

Tampoco se encontró asociación significativa entre presencia de TDM y SM según los distintos criterios, tal como se aprecia en la Tabla 3, incluso después de ajustar por edad y sexo. De hecho, en los tres modelos ajustados, el único factor de riesgo para TDM que persistió fue el ser mujer (ATP-III: OR 7,56, IC 95\% 1,60-35,8; ATP-IIIa: 12,4, IC 95\% 4,61-33,4; IDF: OR 24,4, IC 95\% 7,20-82,4).

El poder del estudio para detectar una asociación entre SM y TDM con OR $\geq 3,5$ fue de $76,6 \%$ si se utiliza la definición de SM de ATP-III, de 80,1\% la del ATP-IIIa y de 80,8\% la del IDF.

\section{DisCUSIÓN}

En el análisis de los resultados de este estudio no fue posible demostrar que existiera algún tipo de

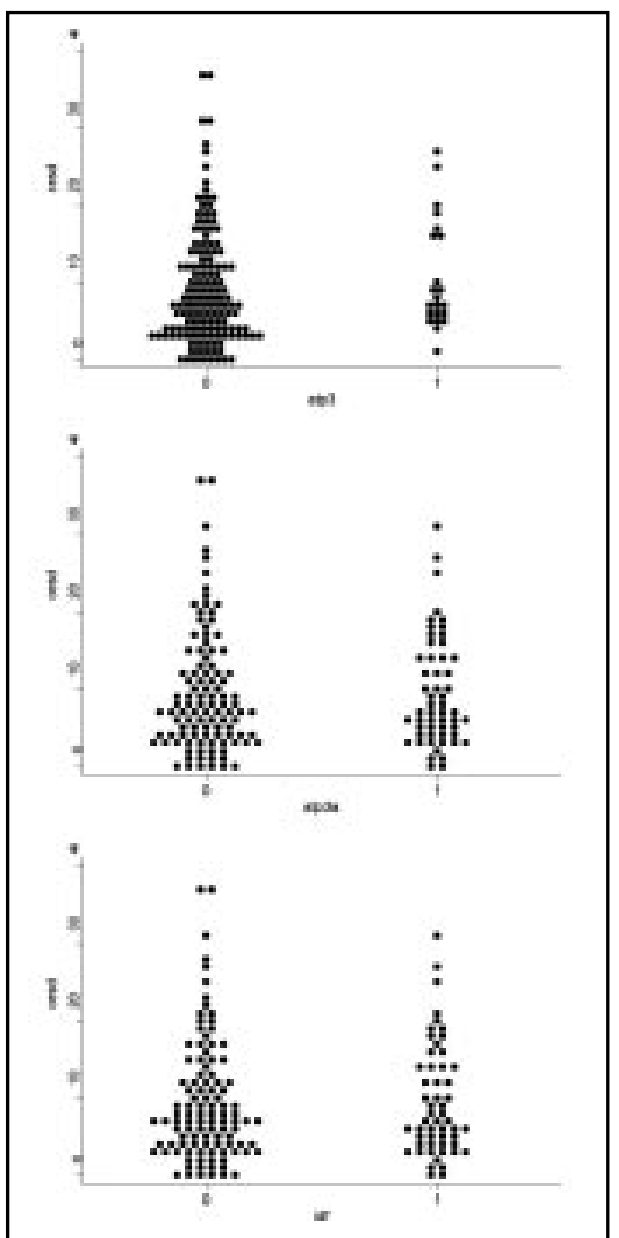

Figura 1. Puntaje en el cuestionario CES-D entre los participantes con y sin síndrome metabólico según las tres definiciones utilizadas. Panel superior: APTIII; panel medio: ATP-IIIa; panel inferior: IDF. 
Tabla 3. Asociación entre riesgo de trastorno depresivo mayor y las tres definiciones de síndrome metabólico existentes

\begin{tabular}{|c|c|c|c|c|c|c|c|c|}
\hline CES-D $\geq 18$ & \multicolumn{2}{|c|}{ SM según ATP-III } & CES-D $\geq 1 \varepsilon$ & \multicolumn{2}{|c|}{ SM según ATP-IIIa } & CES-D $\geq 18$ & \multicolumn{2}{|c|}{ SM según IDF } \\
\hline+ & $\begin{array}{l}+ \\
4\end{array}$ & 20 & + & $\begin{array}{l}+ \\
8\end{array}$ & $\begin{array}{c}- \\
47\end{array}$ & + & $\begin{array}{c}+ \\
9\end{array}$ & - \\
\hline- & 18 & 117 & - & 16 & 88 & - & 15 & 90 \\
\hline \multicolumn{3}{|c|}{$\begin{array}{c}\quad \chi^{2}=0,19, \mathrm{p}=0,663 \\
\text { ORc: } 1,30 \text { (IC 95\% 0,40-4,24) } \\
\text { ORa: } 2,15 \text { (IC 95\% 0,54-8,49) }\end{array}$} & \multicolumn{3}{|c|}{$\begin{array}{c}\chi^{2}=0,02, p=0,888 \\
\text { ORc: } 0,94 \text { (IC 95\% 0,37-2,33) } \\
\text { ORa: } 1,85 \text { (IC } 95 \% 0,58-5,93 \text { ) }\end{array}$} & \multicolumn{3}{|c|}{$\begin{array}{l}\quad \chi^{2}=0,16, p=0,691 \\
\text { ORc: } 1,20 \text { (IC 95\% 0,49-2,95) } \\
\text { ORa: 3,90 (IC 95\% 0,93-13,1) }\end{array}$} \\
\hline
\end{tabular}

ORc: OR crudo; ORa: OR ajustado.

asociación entre la presencia de síndrome metabólico y síntomas depresivos. Esta conclusión se sustenta en que las comparaciones resultantes de las distintas formas de evaluar ambas condiciones (tres para SM, dos para SD) no presentan asociación significativa, y en que la muestra tiene un poder superior a $75 \%$ para detectar OR de 3,5 con las tres definiciones de SM consideradas, según el análisis post hoc de los datos encontrados.

La asociación entre TDM y trastomos cardiovasculares es conocida, donde las personas con el trastomo tienen mayor incidencia de eventos cardiovasculares y peor pronóstico en caso de que el evento no fuese mortal ${ }^{27-29}$. Por otro lado, desde la década 1970-79 se reconocía que el metabolismo de la glucosa estaba alterado en los pacientes que sufnán lo que entonces se conocía como depresión unipolar ${ }^{30}$, lo que llevó al desarmollo de pruebas bioquímicas funcionales que permitieran tener mejor certeza diagnóstica ${ }^{31}$, pero que luego llevaron a pensar que las alteraciones metabólicas de los pacientes con TDM respondían probablemente a una alteración generalizada que incluía resistencia a la insulina ${ }^{32}$.

Dado que una de las cosas que más se han documentado en medicina son los factores de riesgo para eventos cardiovasculares, fue relativamente sencillo pensar en evaluar si la asociación entre TDM y dichos factores de riesgo igualmente existían, incluyendo en este proceso de evaluación al SM, máxime cuando hay una relativamente consistente evidencia que lo hacen plausible biológicamente ${ }^{33,34}$ y a que los pacientes con trastornos del ánimo tienen con más frecuencia hipertensión, obesidad o dislipidemia ${ }^{14,20,35}$.

Los resultados de estudios poblacionales de tipo transversal en distintos países, apoyan la hipótesis sobre la existencia de algún tipo de relación entre SM y TDM ${ }^{15,19,36-38}$, pero no así por el único estudio de cohorte publicado a la fecha ${ }^{39}$. El presente sería, entonces, el primer estudio poblacional de tipo transversal con suficiente poder estadístico que se publica en el que no se apoya la hipótesis sobre la asociación entre SM y TDM.

Es posible que el no encontrar asociación entre TDM y SM esté condicionado parcialmente, por el hecho de que la mitad de la población estudiada apenas llega a los 40 años, en donde la prevalencia de SM es menor, y que la mayoría sean varones, entre quienes la prevalencia de SM y de TDM también es menor, lo que podría diluir la asociación. Sin embargo, la utilización de la regresión logística para ajustar el indicador de asociación, permite controlar la confusión que la edad y el sexo producen sobre este fenómeno, que se refleja en el sustancial incremento que se produce en los OR en los tres modelos explicados, pero sin que lleguen a alcanzar el umbral para ser considerados como significativos desde el punto de vista estadístico.

La aparición del ser mujer como factor de riesgo para SD relevante en los tres modelos no es un hallazgo inesperado, ya que se ha encontrado mayor susceptibilidad de las mujeres a experimentar esta condición, incluyendo la población colombiana ${ }^{2}$, hallazgo que permite apoyar, circunstancialmente, la falta de asociación entre TDM y SM, en la medida que el estudio encontró un factor que era de esperar luego de ajustar por los otros factores conocidos, a más que no se halló asociación entre SM y TDM en el subgrupo de mujeres, tal como se podría esperar del estudio de Räikkönen, Matthews y Kuller, quienes plantean que dadas las características meta- 
bólicas de las mujeres, el SM y el TDM son más factibles en ellas ${ }^{14}$.

Un punto fundamental a tener en cuenta, es que es posible que la asociación entre síndrome metabólico y síntomas depresivos de todas formas exista entre la población estudiada. Un odds ratio de 3,5 implica que, al menos la frecuencia de síntomas depresivos entre quienes tienen síndrome metabólico, sea dos a tres veces más alta que entre quienes no tienen el síndrome, lo que equivaldría a decir que la fuerza de la asociación entre las dos entidades seńa de menor dimensión a la que con el poder de este estudio se puede demostrar ${ }^{40,41}$. En contra de esta posibilidad están las seis formas en que se evaluó la asociación, con tres definiciones reconocidas de SM versus dos maneras de analizar el resultado del CESD, aspectos que fueron aplicados

\section{REFERENCIAS}

1. López AD, Mathers AD, Ezzati M, Jamison DT, MuRRAY CJL (eds). Global burden of disease and risk factors. Washington, The World Bank and Oxford University Press, 2006.

2. Gómez-Restrepo C, Bohórquez A, Pinto D, Gil JFA, Rondón M, Díaz-Granados N. Prevalencia de depresión y factores asociados con ella en la población colombiana. Rev Panam Salud Pública 2004; 16: 378-86.

3. Campo-Arias A, Díaz-Martínez LA, Rueda-Jaimes GE, Cadena LP, HeRnándeZ NL. Validation of Zung's self-rating depression scale among the Colombian general population. Social Behav Personality 2006; 34: 87-94.

4. Perel P, Casas JP, Ortiz Z, Miranda JJ. Noncommunicable diseases and injuries in Latin America and the Caribbean: Time for action. PloS Med 2006; 3: e344.

5. Isomaa B, Almgren P, Tuomi T, Forsen B, Lahti K, NISSEN M ET AL. Cardiovascular morbidity and mortality associated with the metabolic syndrome. Diabetes Care 2001; 24: 683-9.

6. Sattar N, Gaw A, Scherbakova O, Ford I, O'reily DS, HaFFNER SM ET AL. Metabolic syndrome with and without C-reactive protein as a predictor of coronary heart disease and diabetes in the West of Scotland Coronary Prevention Study. Circulation 2003; 108: 414-9.

7. Executive Summary of The Third Report of The National Cholesterol Education Program (NCEP) rigurosamente durante la fase operativa del estudio, lo que se hizo con la intención de reducir al máximo cualquier sesgo de clasificación en ambos aspectos.

En conclusión, la evidencia encontrada en una población de empleados de una Facultad de Medicina indica que, al menos en esta población, no hay asociación entre síndrome metabólico, definido de tres formas, y la presencia de síntomas depresivos evaluados con el CES-D, quizás, la escala de evaluación poblacional más utilizada en el mundo. Con todo, no es posible descartar de plano que esta asociación, hallada en otras investigaciones hechas en otras latitudes y poblaciones, así como las implicaciones clínicas y terapéuticas que se han propuesto a partir de ella ${ }^{13,20}$, razón por la cual es necesario profundizar en este aspecto en la población colombiana.

Expert Panel on Detection, Evaluation, and Treatment of High Blood Cholesterol in Adults (Adult Treatment Panel III). JAMA 2001; 285: 2486-97.

8. Grundy S, Ciefman J, Daniels S, Donato K, Eckel R, FRANKUN B ET AL. Diagnosis and management of the metabolic syndrome. An American Heart Association/National Heart, Lung, and Blood Institute Scientific Statement. Circulation 2005; 112: 2735-52.

9. Alberti KG, Zimmet P, Shaw J. Metabolic syndrome-a new world-wide definition. A Consensus Statement from the International Diabetes Federation. Diabet Med 2006; 23: 469-80.

10. Aschner P. Diabetes trends in Latin America. Diabetes Metab Res Rev 2002; 18 (Suppl 3): S27-31.

11. Vileggas A, Botero JF, Arango K, Arias S, Toro MM. Prevalencia de síndrome metabólico en la población de El Retiro. Iatreia 2003; 16: 291-7.

12. Mendivil C, SierRa I, Pérez C. Valoración del riesgo cardiovascular global y prevalencia de dislipemias según los criterios del NCEP-ATPIII en una población adulta de Bogotá, Colombia. Clin Invest Arterioscl 2004; 16: 99-107.

13. RAMASUBBU R. Insulin resistance: a metabolic link between depressive disorder and atherosclemotic vascular diseases. Med Hypotheses 2002; 59: 537-51.

14. RäIKKÖNEN K, MATTHEWS KA, KULER LH. The relationship between psychological risk attributes and the metabolic syndrome in healthy women: antecedent or consequence? Metabolism 2002; 51: 1573-7.

15. Kinder LS, Carnethon MR, Palaniappan LP, King AC, FoRTMANn SP. Depression and the metabolic syn- 
drome in young adults: findings from the Thind National Health and Nutrition Examination Survey. Psychosom Med 2004; 66: 316-22.

16. LetT HS, Bumenthal JA, Babyak MA, Sherwood A, Strauman T, Robins C ET al. Depression as a risk factor for coronary artery disease: evidence, mechanisms, and treatment. Psychosom Med 2004; 66: 305-15.

17. Petrlova B, Rosolova H, Hess Z, Podlpiny J, Simon J. Depressive disorders and the metabolic syndrome of insulin resistance. Semin Vasc Med 2004; 4: 161-5.

18. Dixon JB, Anderson M, Cameron-Smith D, O’Brien PE. Sustained weight loss in obese subjects has benefits that are independent of attained weight. Obes Res 2004; 12: 1895-902.

19. McCaffery JM, Niaura R, Todaro JF, Swan GE, CARMEL D. Depressive symptoms and metabolic risk in adult male twins enrolled in the National Heart, Lung, and Blood Institute twin study. Psychosom Med 2003; 65: 490-7.

20. McElroy SL, Kotwal R, Malhotra S, Nelson eB, KeCK PE, Nemeroff CB. Are mood disorders and obesity related? A review for the mental health professional. J Clin Psychiatry 2004; 65: 634-51.

21. Pickering TG. Recommendations for the use of home (self) and ambulatory blood pressure monitoring. American Society of Hypertension $\mathrm{Ad}$ Hoc Panel. Am J Hypertens 1996; 9: 1-11.

22. Marks GC, Habickt JP, Muewer WH. Reliability, dependability, and precision of anthropometric measurements, The Second National Health and Nutrition Examination Survey 1976-1980. Am J Epidemiol 1989; 130: 578-87.

23. Moreno-Altamirano A, López-Moreno S, CorchoBerdugo A. Principales medidas en epidemiología. Salud Púb Méx 2000; 42: 337-48.

24. WiLCoxon F. Individual comparisons by ranking methods. Biometrics 1945; 1: 80-3.

25. Hernández B, Velasco-Mondragón HE. Encuestas transversales. Salud Púb Méx 2000; 42: 447-55.

26. STATACORP. Stata statistical software: release 9. College Station, StataCorp LP, 2005.

27. Anda R, Wimiamson D, Jones D, Macera C, Eaker E, Glassman A ET AL. Depressed affect, hopelessness, and the risk of ischemic heart disease in a cohort of U.S. adults. Epidemiology 1993; 4: 285-94.

28. Sesso HD, Kawachi I, Vokonas PS, Sparrow D. Depression and the risk of comonary heart disease in the Normative Aging Study. Am J Cardiol 1998; 82: 851-6.

29. Penninx BW, Beekman AT, Honig A, Degg DJ, Schoevers RA et AL. Depression and cardiac morta- lity: results form a community-based longitudinal study. Arch Gen Psychiatry 2001; 58: 221-7.

30. Wright JH, Jacicin JJ, Radin NS, Beil RA. Glucose metabolism in unipolar depression. Br J Psychiatry 1978; 132: 386-93.

31. Koslow SH, Stokes PE, Mendels J, Ramsey A, Casper R. Insulin tolerance test: human growth hormone response and insulin resistance in primary unipolar depressed, bipolar depressed and control patients. Psychol Med 1982; 12: 45-55.

32. Winokur A, Maislin G, Phimps JL, Amsterdam JD. Insulin resistance after oral glucose tolerance testing in patients with major depression. Am J Psychiatr 1988; 145: 325-30.

33. Okamura F, Tashiro A, Utumi A, Imai T, Suchi T, TAMURA $S$ ET AL. Insulin resistance in patients with depression and its changes during the clinical course of depression: minimal model analysis. Metabolism 2000; 49: 1255-60.

34. Kopf D, Westphal S, Luley CW, Ritter S, Giues M, WeBER-Hamann B ET AL. Lipid metabolism and insulin resistance in depressed patients. Significance of weight, hypencortisolism, and antidepressant treatment. J Clin Psychopharmacol 2004; 24: 527-31.

35. WidDES JE, Marcus MD, Fagioun A. Obesity in patients with bipolar disonder. a biopsychosocial-behavioral model. J Clin Psychiatry 2006; 67: 904-15.

36. Pannier B, Thomas F, Eschwege E, Bean K, Benetos A, LEOCMACH Y, ET AL. Cardiovascular risk markers associated with the metabolic syndrome in a large French population: the «YYMFONIE» study. Diabetes Metab 2006; 32: 467-74.

37. Podipny J, Hess Z. Deprese, metabolicky syndrom a diabetes mellitus. Interní Med 2006; 7-8: 348-51.

38. Lawlor DA, Smith GD, EвRAhim S. Association between insulin resistance with depression: cross sectional findings from the British Women's Heart and Health Study. BMJ 2003; 327: 1383-4.

39. Herva A, Rasanen P, Miettunen J, Timonen M, Laksy K, Vejola J et al. Co-occurrence of metabolic syndrome with depression and anxiety in young adults: the Northern Finland 1966 Birth Cohort Study. Psychosom Med 2006; 68: 213-6.

40. Streiner DL. Sample size and power in psychiatric Research. Can J Psychiatry 1990; 35: 616-20.

41. Houle TT, Penzien DB, Houle CK. Statistical power and sample size estimation for headache research: an overview and power calculation tools. Headache 2005; 45: 414-8. 